\title{
Psychometric evaluation of the Participation and Activity Inventory for Children and Youth (PAI-CY) 0-2 years with visual impairment
}

\author{
Ellen B. M. Elsman ${ }^{1,3}$ (D) Ruth M. A. van Nispen ${ }^{1}$ (D) Gerardus H. M. B. van Rens ${ }^{1,2}$ (D)
}

Accepted: 18 October 2019 / Published online: 31 October 2019

(c) The Author(s) 2019

\begin{abstract}
Purpose To identify and monitor the developmental and participation needs of visually impaired (VI) children, the Participation and Activity Inventory for Children and Youth (PAI-CY) has recently been developed involving end-users as stakeholders. The aim was to investigate psychometric properties of the PAI-CY for children between 0 and 2 years.

Methods Responses from 115 parents were included in item analyses, after which a combination of classical test theory and item response theory (IRT) was used. Internal consistency, known-group validity, and test-retest reliability at item and scale level were investigated.

Results After deleting four items, the PAI-CY met IRT assumptions, i.e., unidimensionality, local independence, and monotonicity, and satisfactory model fit was obtained. Participants with more severe VI and comorbidity scored significantly worse than those with less severe VI and without comorbidity, supporting known-group validity. Satisfactory internal consistency and test-retest reliability were obtained (Cronbach's alpha 0.95, kappa 0.60-0.91, ICC 0.920).

Conclusions The PAI-CY 0-2 years has acceptable psychometric properties and can be used to systematically assess and monitor developmental and participation needs of very young children with VI from parents' perspectives in low vision practice and research. Confirmation of psychometric properties is necessary, possibly facilitating further item reduction, increased precision, and improved user-friendliness.
\end{abstract}

Keywords Validation $\cdot$ Psychometrics $\cdot$ Children $\cdot$ Visual impairment $\cdot$ Item response theory $\cdot$ Participation

\section{Introduction}

Although the prevalence of childhood visual impairment (VI) is low [1], it has lifelong and far-reaching implications, for both children and their parents. According to parents of children with VI in the age band of 0-2 years and professionals with expertise in VI for this particular age group, sensory and general developmental issues related to attachment and well-being were among the most important concerns [2].

Ellen B. M. Elsman

e.elsman@amsterdamumc.nl

1 Department of Ophthalmology, Amsterdam Public Health Research Institute, Amsterdam UMC, Vrije Universiteit Amsterdam, De Boelelaan 1117, Amsterdam, The Netherlands

2 Department of Ophthalmology, Elkerliek Hospital, Wesselmanlaan 25, 5707 HA Helmond, The Netherlands

3 Amsterdam UMC, VU University Medical Center, PK4X191, PO Box 7057, 1007 MB Amsterdam, The Netherlands
In the Netherlands, low vision services offer guidance such as developmental and behavioral interventions to overcome challenges related to vision loss. One of the most important outcomes of low vision services in children with visual impairment is participation, which for young children usually takes place in the family context [3]. In order to structure the process of identifying needs of children and their parents, the Participation and Activity Inventory for Children and Youth (PAI-CY) was recently developed involving end-users as stakeholders [2]. To aid interpretation, four different questionnaires were developed to reflect the developmental age bands of children as set by the World Health Organization (WHO). The PAI-CY should lead to a patient-based assessment of the impact of VI on functioning and participation and should initiate shared decision-making about interventions needed. Results from a pilot study showed that parents were mostly satisfied with the PAI-CY, whereas professionals suggested some changes which were incorporated in the next version [4]. The current study aims 
to investigate the psychometric properties of the PAI-CY $0-2$ years.

\section{Methods}

\section{Participants and procedure}

Parents/caretakers (parents for brevity) of children aged 0-2 years registered at two Dutch low vision services were invited to participate. Parents who agreed to participate completed questions regarding socio-demographic and clinical information, the PAI-CY 0-2 years, and an evaluation form. Two weeks later [5], parents completed a retest. Although it should be noted that the very young age of children might result in less accurate data, ophthalmic diagnosis, visual acuity, and visual field of children were retrieved from patient files; missing values were complemented with self-reported data from parents $(n=10)$. VI was divided by five levels based on the better seeing eye and corresponding to the WHO criteria [6].

\section{PAI-CY 0-2 years}

The preliminary version of the PAI-CY 0-2 years comprises 31 items categorized into seven domains (for descriptive purposes only, in order to provide contextual meaning): attachment, stimulus processing, visual attention, orientation, play, mobility, and communication that were informed by qualitative data from parents and concept-mapping workshops with professionals [2]. Each item is scored on a 4-point Likert scale with the following response options: not difficult (1), slightly difficult (2), very difficult (3), and impossible (4). The response option not applicable was treated as a missing value.

\section{Statistical analyses}

Item analyses were conducted by examining missing responses and response category distributions. Items with missing scores $>20 \%$ were considered for elimination, as were items with $>70 \%$ of the respondents endorsing the first or last response category (i.e., floor or ceiling effects) and items having no answer in one of the response categories. Items showing inter-item correlations $>0.8$, indicating potential redundancy, were also considered for elimination, as were items with an item-total correlation $<0.3$. Cronbach's alpha was calculated to evaluate internal consistency reliability.

An item response theory (IRT) model was subsequently applied. Items violating basic assumptions were considered for elimination [7-10]. Unidimensionality [11] was assessed by performing an eigenvalue decomposition on the matrix of robust (Spearman) correlations between the items. A difference approximation to the second-order derivatives along the eigenvalue curve (scree plot) was calculated. This acceleration approximation indicates points of abrupt change along the eigenvalue curve, and the number of eigenvalues before the point with the most abrupt change (the point with the maximum acceleration value) represents the number of latent dimensions that dominate the information content [7]. Subsequently, a principal component analyses (PCA) was performed to proxy if all items load on a single component (where the component is taken as a proxy for the latent trait). Magnitude of principal components was checked. Item pairs with excess covariation $(>0.25)$, signaling local dependence, were flagged. Monotonicity was assessed using Mokken scale analyses. The resulting graphs were visually inspected, and a Loevinger $\mathrm{H}$ coefficient was calculated to assess scalability ( $<0.3$ was considered unsatisfactory) [8-10]. The graded response model (GRM) was used [12, 13]; a full model was compared with a constrained model $[11,14,15]$ nested within the full model with equal slope parameters across items. The models were compared using a likelihood ratio test (LRT). Relevant model fit indices were checked [16, 17]. Individual item performance was examined by assessing item fit using the $X^{2}$ statistic. In addition, item and test information curves [11, 18, 19] were computed as well as an item-person map [20].

Known-group validity [5] was investigated using independent samples $t$ tests and ANOVAs using post hoc Tukey tests for the following characteristics: gender, comorbidity, age (median split: $\leq 20$ months vs. $>20$ months), and level of VI according to the WHO criteria [6]. Test-retest reliability at item level was investigated using weighted kappa and percentage agreement $[5,21,22]$ The intraclass correlation coefficient (ICC) for thetas calculated on test-retest data was based on absolute agreement in a two-way mixed-effects model.

All statistical analyses were conducted in R-Studio [23] and SPSS version 22 [24].

\section{Results}

Of an estimated 290 invited parents, 131 provided written informed consent to participate and completed the first questionnaire (45\%). Data from participants $>25 \%$ missing responses on the PAI-CY $0-2$ years $(n=14)$ or children $>2$ years $(n=2)$ were excluded from the analyses. Table 1 presents characteristics of participants $(n=115)$. Out of the 115 participants, 54 had complete data on the PAI-CY $0-2$ years, 45 respondents had one to three missing items, while nine respondents had $>5$ missing items. Items were 
Table 1 Socio-demographic and clinical characteristics of participants $(n=115)$

\begin{tabular}{ll}
\hline Age in months, mean \pm SD (range) & $20.91 \pm 7.07$ (7-35) \\
Male gender, $n(\%)$ & $74(64.3)$ \\
Severity of VI [5], $n(\%)$ & $18(15.7)$ \\
No VI: logMAR $\leq 0.3$ & $14(12.2)$ \\
Mild VI: logMAR $0.31-0.52$ & $46(40.0)$ \\
Moderate VI: logMAR 0.53-1.00 & $12(10.4)$ \\
Severe VI: logMAR 1.01-1.30 & $15(13.0)$ \\
Blind: logMAR $\geq 1.31$ or visual field $\leq 10^{\circ}$ & $10(8.7)$ \\
Unknown & $65(56.5)$ \\
Comorbidity, $n(\%)$ & \\
Parent who completed the questionnaire, $n(\%)$ & $90(78.3)$ \\
Mother & $13(11.3)$ \\
Father & $11(9.6)$ \\
Mother and father together & $1(0.9)$ \\
Caretaker & \\
Nationality parent, $n(\%)$ & $110(95.7)$ \\
Dutch & $5(4.3)$ \\
Other & $13.54 \pm 2.72(0-16)$ \\
Education in years parent, mean \pm SD (range) & \\
Financial situation parent, $n(\%)$ & $65(56.5)$ \\
Usually enough money & $26(22.6)$ \\
Just enough money & $5(4.3)$ \\
Not enough money & $19(16.5)$ \\
No answer & \\
\hline
\end{tabular}

missing at random; there were no indications for acquiescence bias. Over $90 \%$ of the respondents were neutral to very positive about various aspects of the PAI-CY $0-2$ years (Fig. 1), and no suggestions for improvements were made more than once. Self-reported administration time (including questions on demographic and clinical characteristics) was
$17 \pm 7$ (range 5-40, median 15$) \mathrm{min}$. The retest $(n=108)$ was completed after a mean of $30.5 \pm 26.4$ (range 11-181, median 19) days.

The items "reacting to (sudden) sounds" and "entertaining alone" were deleted because of low factor loadings, low information, and low scalability coefficients. "Looking at a particular item nearby" and "following a toy or person with the eyes" were deleted because of local dependence with too many other items. Furthermore, omitting these items was considered not to violate content validity, because they were thought not to be suitable for the target population (entertaining alone) or because of similarity to other items.

None of the items had disturbing amounts of missing responses according to our cut-off criterion, whereas three items demonstrated floor effects. In general, the fourth answer category was infrequently endorsed, but collapsing answer category 3 and 4 led to questionable unidimensionality, as the relative increase of the explained variance of the second factor was limited compared to the first factor. Therefore, the response categories of only a few items were collapsed (indicated in bold in Table 2). Four item pairs showed high inter-item correlations (Table 2), whereas none of the items had item-total correlations $<0.3$. Cronbach's alpha was 0.95 .

The assumptions for IRT seemed to hold for most items (Table 2 shows items violating assumptions). The items represented a unidimensional model; a one-factor model explained $48 \%$ of the variance and mostly yielded high component loadings $(>0.45)$. A two-factor solution added $10 \%$ explained variance. The ratio of 4.8 between the first and second factor is higher than the required minimum of 4 [25]. Out of 351 possible item pairs, three item pairs violated the local independence assumption. One item violated the monotonicity assumption and one item had an insufficient
Fig. 1 Evaluation of the PAI-CY 0-2 years by parents $(n=115)$

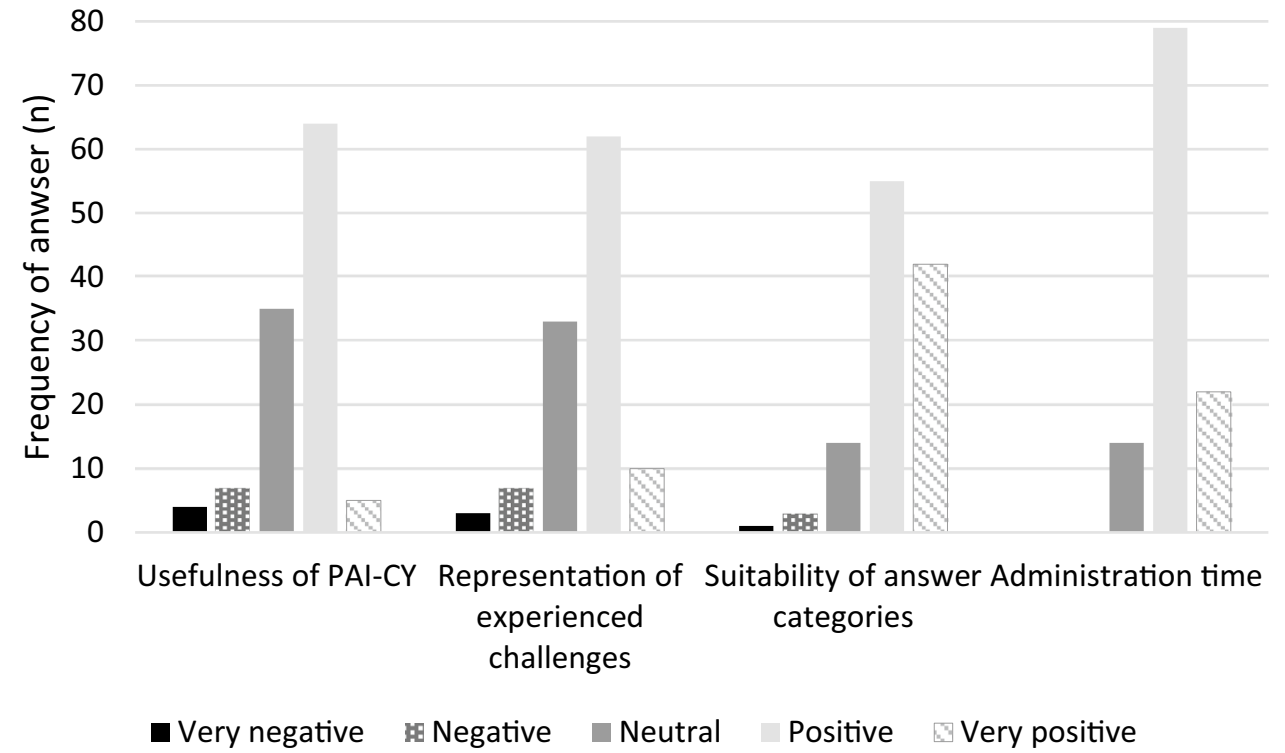




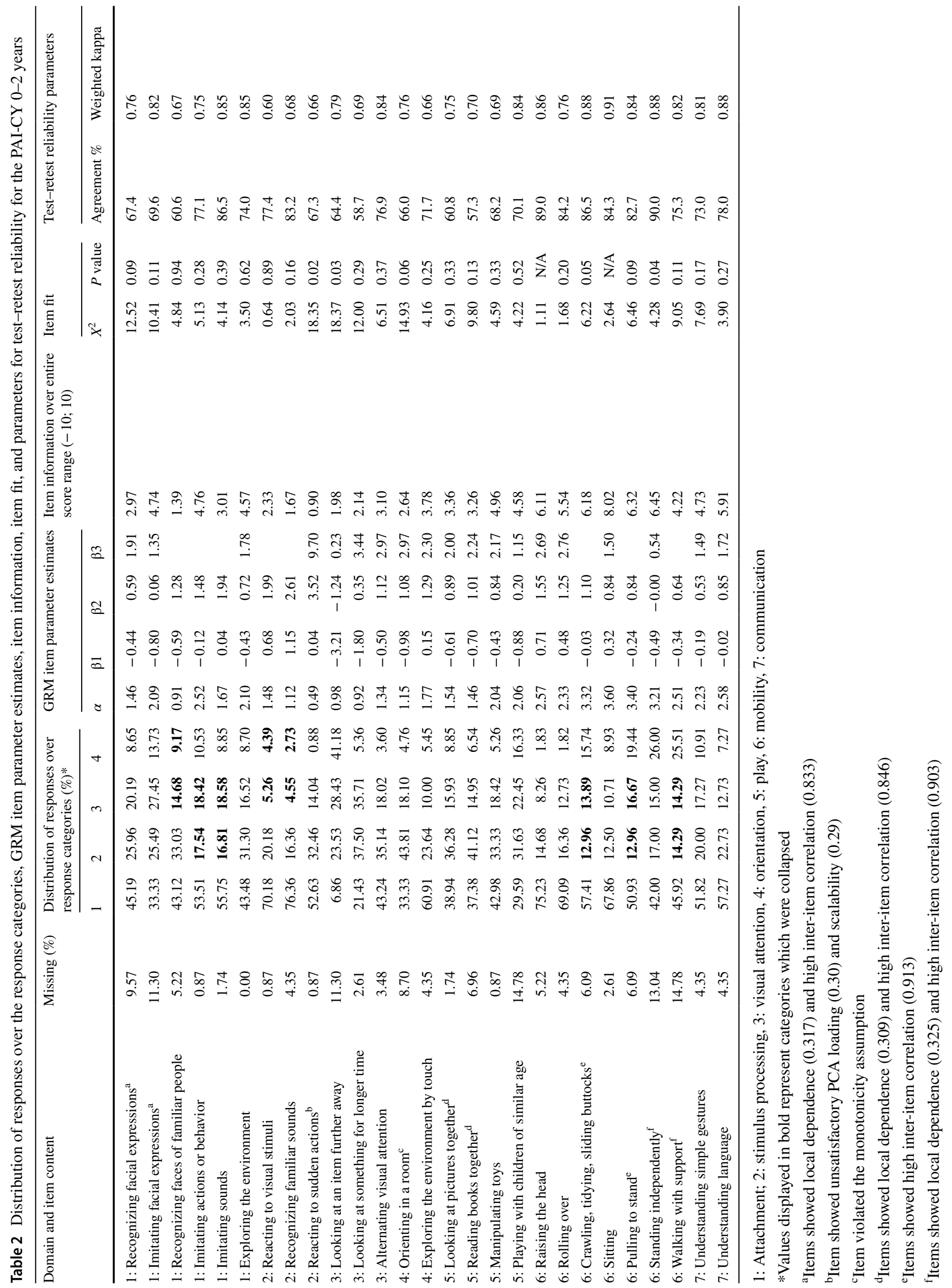


scalability coefficient. It was decided not to delete these items because of content relevance.

The full GRM outperformed the constrained model (LRT $=116.42, \mathrm{df}=26, p<0.001)$, and model fit approached satisfactory values $(\mathrm{RMSEA}=0.064, \mathrm{SRMR}=0.099$, $\mathrm{TLI}=0.965$, CFI $=0.968$ ). Item parameters, information, and fit statistics are displayed in Table 2. We confirmed the validity of the IRT parameters by examining the differences in test-retest parameters, which were on average small $(\alpha: 0.53 \pm 0.48, \beta 1: 0.21 \pm 0.17, \beta 2: 0.29 \pm 0.27, \beta 3$ : $0.43 \pm 0.32$ ); note that test and retest data are highly correlated $(r=0.92)$. Despite the fact that some items provided little information, all items were maintained, because removing the item with lowest information ("reacting to sudden actions") resulted in more violations of assumptions for the remaining items. Difficulty of items matched respondents' ability (Fig. 2).

Regarding known-group validity, participants with comorbidity scored worse on the PAI-CY than those without comorbidity ( $p<0.001$, respectively). Moreover, participants with severe VI or blindness scored significantly worse than participants with no VI $(p=0.030$ and $p=0.023$, respectively). A trend for worsening scores with increasing severity of VI was observed. No significant difference in PAI-CY scores was found for age and sex (Fig. 3).

Most items showed satisfactory test-retest reliability (Table 2), although for two items ("looking at something for a longer period of time" and "reading books together") agreement was $<60 \%$ and for one item ("reacting to visual stimuli") weighted kappa was 0.6. The ICC between test and retest data was 0.920 (95\% confidence interval $0.880-0.946$ ).

\section{Discussion}

With these acceptable psychometric properties, the PAICY 0-2 years should be useful in low vision services, in which the perspectives from parents of very young children with VI can now systematically be assessed. After deleting 4 items, the remaining 27 items showed satisfactory model fit and a unidimensional scale measuring developmental and

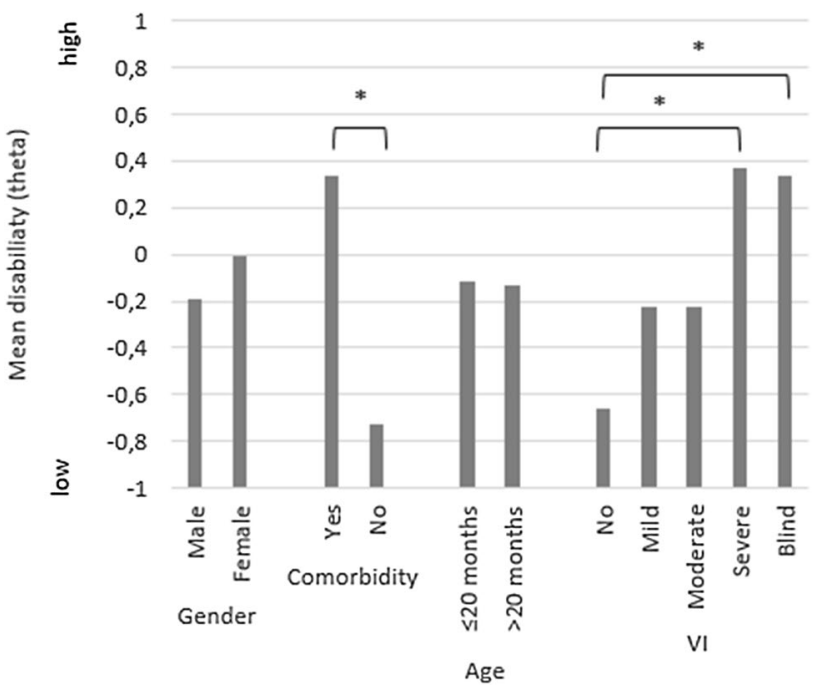

Fig. 3 Mean disability (theta) by gender, presence of comorbidity, age, and severity of VI. ${ }^{*} p<0.05$

participation needs. Although we observed some violations in IRT assumptions, it was decided not to delete any items at this stage. Furthermore, removal of the worst performing item ("reacting to sudden actions") caused more violations in assumptions and worsened model fit. The PAI-CY 0-2 year seems able to discriminate between participants with varying levels of clinical conditions, i.e., comorbidity and degree of VI.

Not many instruments focusing on functioning, participation, and/or quality of life are available for children this young age. Instruments for children with disabilities [26] or visual impairment are even more scarce [27, 28]. To our knowledge, the Children's Visual Function Questionnaire (CVFQ) is currently the only instrument with a version for children aged below 3 years [29]. The CVFQ was developed to measure vision-related quality of life with domains related to competence, personality, family impact, and treatment difficulty imposed by specific eye conditions and might be complementary to the PAI-CY 0-2 years.

The limited sample size of our study was unavoidable; visual disabilities in early childhood are rare among the 17
Fig. 2 Item-person map of the PAI-CY 0-2 years

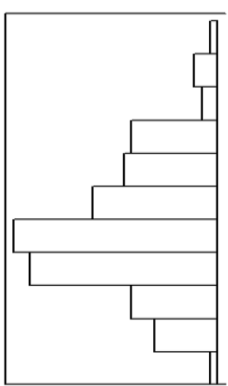

Frequency of person

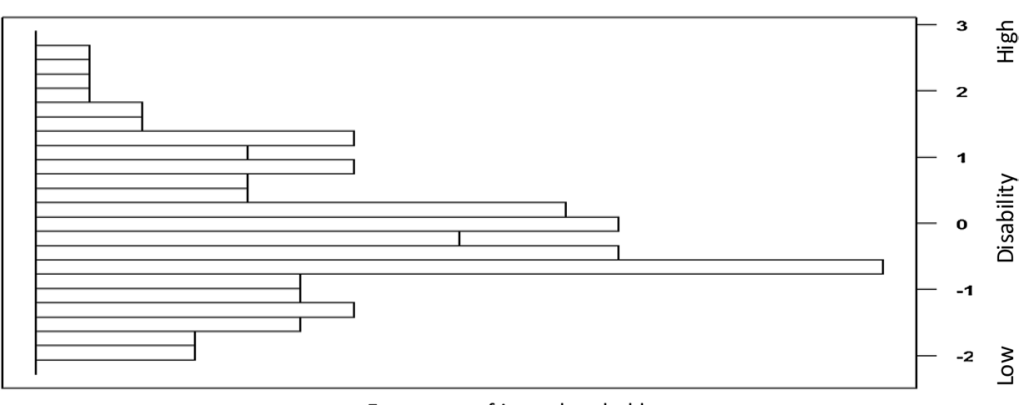

Frequency of item thresholds 
million Dutch inhabitants. We took a conservative approach because of the small sample size (only four items were deleted), using less stringent criteria for item removal in order not to compromise face and content validity. Deleting potentially relevant and informative items prior to the availability of larger samples could be counterproductive in the long term. The planned use of the PAI-CY 0-2 years by Dutch low vision services and in research as a patientreported outcome measure enables confirming its psychometric properties. New applications of IRT for small sample sizes, using longitudinal data, should be considered [30]. This might facilitate further item reduction, increase precision, and improve user-friendliness. Moreover, feasibility and acceptability of the questionnaire to respondents and professionals in clinical care should be monitored.

Acknowledgements We greatly thank all participating parents for their contributions. Funding for this research was provided by Royal Dutch Visio, who had no role in the design, conduct or outcomes of this study.

Funding This study was funded by Royal Dutch Visio/Novum.

\section{Compliance with ethical standards}

Conflict of interest The authors declare that they have no conflict of interest.

Ethical approval The study protocol was approved by the Medical Ethical Committee of the VU University Medical Center, Amsterdam, the Netherlands. All procedures performed in the study involving human participants were in accordance with the ethical standards of the institutional and/or national research committee and with the 1964 Helsinki declaration and its later amendments or comparable ethical standards.

Informed consent Informed consent was obtained from all individual participants included in the study.

Open Access This article is distributed under the terms of the Creative Commons Attribution 4.0 International License (http://creativeco mmons.org/licenses/by/4.0/), which permits unrestricted use, distribution, and reproduction in any medium, provided you give appropriate credit to the original author(s) and the source, provide a link to the Creative Commons license, and indicate if changes were made.

\section{References}

1. Bourne, R. R. A., Flaxman, S. R., Braithwaite, T., Cicinelli, M. V., Das, A., Jonas, J. B., et al. (2017). Magnitude, temporal trends, and projections of the global prevalence of blindness and distance and near vision impairment: A systematic review and meta-analysis. The Lancet Global Health, 5(9), e888-e897. https://doi.org/10.1016/S2214-109X(17)30293-0.

2. Rainey, L., Elsman, E. B. M., van Nispen, R. M. A., van Leeuwen, L. M., \& van Rens, G. (2016). Comprehending the impact of low vision on the lives of children and adolescents: A qualitative approach. Quality of Life Research, 25(10), 2633-2643. https://doi.org/10.1007/s11136-016-1292-8.
3. McConachie, H., Colver, A., Forsyth, R., Jarvis, S., \& Parkinson, K. (2006). Participation of disabled children: How should it be characterised and measured? Disability and Rehabilitation, 28(18), 1157-1164.

4. Elsman, E. B. M., van Nispen, R. M. A., \& van Rens, G. (2017). Feasibility of the participation and activity inventory for children and youth (PAI-CY) and young adults (PAI-YA) with a visual impairment: A pilot study. Health and Quality Life Outcomes, 15(1), 98. https://doi.org/10.1186/s12955-017-0677-x.

5. De Vet, H. C. W., Terwee, C. B., Mokkink, L. B., \& Knol, D. L. (2011). Measurement in medicine: A practical guide. New York: Cambridge University Press.

6. WHO. (1994). ICD-10: International statistical classification of diseases and related health problems, 10th revision. Geneva: WHO.

7. Raîche, G., Walls, T. A., Magis, D., Riopel, M., \& Blais, J.-G. (2013). Non-graphical solutions for Cattell's scree test. Methodology, 9, 23-29.

8. Loevinger, J. (1948). The technic of homogeneous tests compared with some aspects of scale analysis and factor analysis. Psychological Bulletin, 45(6), 507-529.

9. Meijer, R. R., \& Baneke, J. J. (2004). Analyzing psychopathology items: A case for nonparametric item response theory modeling. Psychological Methods, 9(3), 354-368. https://doi. org/10.1037/1082-989X.9.3.354.

10. Sijtsma, K., Meijer, R. R., \& van der Ark, L. A. (2011). Mokken scale analysis as time goes by an update for scaling practitioners. Personality and Individual Differences, 50(1), 31-37. https://doi. org/10.1016/j.paid.2010.08.016.

11. Edelen, M. O., \& Reeve, B. B. (2007). Applying item response theory (IRT) modeling to questionnaire development, evaluation, and refinement. Quality of Life Research, 16(Suppl 1), 5-18. https ://doi.org/10.1007/s11136-007-9198-0.

12. Samejima, F. (1969). Estimation of latent ability using a response pattern of graded scores. Psychometrika, 34(4p2), 1-\&.

13. Samejima, F. (1997). Graded response model. In W. Van der Linden \& R. K. Hambleton (Eds.), Handbook of modern item response theory (pp. 85-100). New York: Springer.

14. Bond, T. G., \& Fox, C. M. (2007). Applying the Rasch modelFundamental measurement in the human sciences (2nd ed.). Mahwah: Lawrence Erlbaum Associates Publishers.

15. Rizopoulos, D. (2006). 1tm: An R package for latent variable modeling and item response theory analyses. Journal of Statistical Software, 17(5), 1-25.

16. Chalmers, R. P. (2012). mirt: A multidimensional item response theory package for the R environment. Journal of Statistical Software, 48(6), 1-29.

17. Hu, L. T., \& Bentler, P. M. (1999). Cutoff criteria for fit indexes in covariance structure analysis: Conventional criteria versus new alternatives. Structural Equation Modeling, 6(1), 1-55. https://doi. org/10.1080/10705519909540118.

18. Bock, R. D. (1972). Estimating item parameters and latent ability when responses are scored in two or more nominal categories. Psychometrika, 37(1), 29-51.

19. Yen, W. M. (1981). Using simulation results to choose a latent trait model. Applied Psychological Measurement, 5(2), 245-262.

20. Irribarra, T. D., Freund, R. (2014). Wright Map: IRT item-person map with ConQuest integration.

21. Altman, D. G. (1991). Practical statistics for medical research. London: Chapman and Hall.

22. Singh, A. S., Vik, F. N., Chinapaw, M. J. M., Uijtdewilligen, L., Verloigne, M., Fernandez-Alvira, J. M., et al. (2011). Testretest reliability and construct validity of the ENERGY-child questionnaire on energy balance-related behaviours and their potential determinants: The ENERGY-project. International 
Journal of Behavioral Nutrition and Physical Activity. https:// doi.org/10.1186/1479-5868-8-136.

23. R Core Team. (2017). R: A language and environment for statistical computing. Vienna, Austria. https://www.R-project.org/: R Core Team. R Foundation for Statistical Computing.

24. IBM. (2013). SPSS Statistics for Windows, Version 22.0. Armonk, NY: IBM Corp.

25. Reeve, B. B., Hays, R. D., Bjorner, J. B., Cook, K. F., Crane, P. K., Teresi, J. A., et al. (2007). Psychometric evaluation and calibration of health-related quality of life item banks: Plans for the patientreported outcomes measurement information system (PROMIS). Medical Care, 45(5), S22-S31.

26. Rainey, L., van Nispen, R., van der Zee, C., \& van Rens, G. (2014). Measurement properties of questionnaires assessing participation in children and adolescents with a disability: A systematic review. Quality of Life Research, 23(10), 2793-2808. https:// doi.org/10.1007/s11136-014-0743-3.

27. Tadic, V., Hogan, A., Sobti, N., Knowles, R. L., \& Rahi, J. S. (2013). Patient-reported outcome measures (PROMs) in paediatric ophthalmology: A systematic review. British Journal of Ophthalmology, 97(11), 1369-1381. https://doi.org/10.1136/bjophthalm ol-2013-303350.
28. Tadic, V., \& Rahi, J. S. (2017). One size doesn't fit all: Time to revisit patient-reported outcome measures (PROMs) in paediatric ophthalmology? Eye (London), 31(4), 511-518. https://doi. org/10.1038/eye.2016.316.

29. Birch, E. E., Cheng, C. S., \& Felius, J. (2007). Validity and reliability of the Children's Visual Function Questionnaire (CVFQ). Journal of American Association for Pediatric Ophthalmology and Strabismus, 11(5), 473-479.

30. Houts, C. R., Morlock, R., Blum, S. I., Edwards, M. C., \& Wirth, R. J. (2018). Scale development with small samples: A new application of longitudinal item response theory. Quality of Life Research, 27(7), 1721-1734. https://doi.org/10.1007/s1113 6-018-1801-z

Publisher's Note Springer Nature remains neutral with regard to jurisdictional claims in published maps and institutional affiliations. 